\title{
Assessment of the needs of vulnerable youth populations in post-conflict Liberia
}

\author{
EJ Levey1, CPC Borba1,2, BL Harris³, JR Carney', S Domínguez', EKS Wang1,2, R Boxill', DC Henderson ${ }^{1,2}$ \\ ${ }^{1}$ The Chester M. Pierce, MD Division of Global Psychiatry, Massachusetts General Hospital, Boston, MA, USA \\ 2Harvard Medical School, Boston, MA, USA \\ ${ }^{3}$ A.M. Dogliotti College of Medicine, University of Liberia, Monrovia, Liberia
}

\begin{abstract}
Objective: The study examined key informants' perceptions of the emotional impact of traumatic events, major problems, functional limitations and appropriate treatment settings for children, adolescents, and young adults in post-conflict Liberia. Method: This research is a based on cross-sectional survey conducted between March 30, 2009 and April 30, 2009 in Liberia with 171 local key Liberian informants. Analysis was conducted using mixed methods. The findings we will report were collected in the qualitative portion of the survey. Results: We found that while different interventions were preferred for different types of young people, some interventions were mentioned for all youth and by all age and gender groups of key informants. These included counseling, education, and skills training. Also frequently chosen were housing, community reintegration, recreation, and medical care. In general, key informants reported similar concerns regardless of their ages or genders. Notable exceptions to this were in interventions for youth who joined fighting forces. Men over 50 were the only ones to recommend apology and reparations. Similarly, in recommendations for criminals and violent youth, a number of men mentioned prison, whereas the women did not. Conclusion: Our findings suggest that the needs of post-conflict Liberian youth span a variety of domains, including physical, emotional, medical, psychological, and educational. These findings can be used to guide the development of treatment programs for these young people.
\end{abstract}

Keywords: Post-Conflict Liberia; Mental Health Needs Assessment; Children and Adolescents

Received: $26-10-2012$

Accepted: 27-1 1-2012

doi: http://dx.doi.org/10.4314/ajpsy.v16i5.47

\section{Introduction}

The Republic of Liberia lies on the horn of West Africa. From the most recent census conducted in 2008, it has a population of approximately 3.5 million people. ${ }^{1}$ Between 1989 and 2003, the country experienced a brutal civil war characterized by ethnic killings, sexual violence and the use of child soldiers. The war displaced a huge proportion of the population, destroyed the productive capacity and physical infrastructure of the country, and eroded family and community ties. ${ }^{2}$

Post-conflict societies invariably struggle with reestablishing order while at the same time facing major health

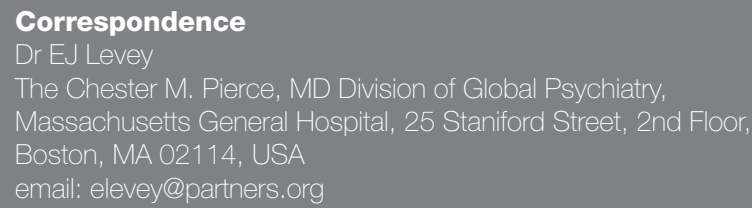

consequences of the trauma experienced by their populations. In addition to medical issues, these consequences frequently include mental illnesses such as post-traumatic stress, mood, and substance use disorders. There is a small body of research that has looked at mental health issues in postconflict societies. Data has been gathered from other African countries including Uganda, Rwanda, and Sierra Leone.

A meta-analysis of 17 studies of children in post-conflict societies from around the world found that PTSD, depression, and anxiety were all increased in this population. ${ }^{3}$ A study done in Rwanda used key informant interviews to identify protective factors associated with resilience in children of families affected by genocide and HIV. The main factors identified were perseverance, self esteem, family unity, good parenting, and community cohesion. ${ }^{4}$ In Liberia, a survey of households and clinics in rural Nimba county found that while most individuals had access to malaria and HIV care, only $12 \%$ could access mental health care. ${ }^{5}$ 
A few researchers have sought to understand the factors that affect mental health outcomes in children exposed to the violence of war. Some suggest that there are factors separate from the trauma itself that affect outcomes because there is a significant variance that cannot be explained by differences in trauma alone. A model has been proposed in which daily stressors mediate the effects of exposure to violence on mental health. 6 Degree of stigma experienced by former child soldiers also has a significant impact on mental health. ${ }^{6,7}$ Another study examined the sense of coherence among children and adolescents who lived through the war in Croatia. Sense of coherence is the extent to which people are confident that their environment is predictable and that things will work out as well as can reasonably be expected. ${ }^{8}$ The study sought to determine which factors influenced the degree to which participants had a sense of coherence about their experiences and whether that correlated with their ability to respond to stressors. Childhood stability, acceptance of own ethnic identity, management of uncertainty, and the meanings of work emerged as the responses and resources that may influence sense of coherence. ${ }^{9}$

A few studies have evaluated the effectiveness of brief educational interventions to increase resiliency among children in post-conflict settings. In northern Uganda, a schoolbased educational intervention was designed to target communities that experienced a high rate of displacement in the recent conflict. Findings demonstrated improved school performance and resilience at 12-month follow-up compared with children who did not experience the intervention. ${ }^{10}$ Children displaced by conflict in Sierra Leone were interviewed before and after the Rapid-Ed intervention, designed to educate and target trauma symptoms. Findings suggest intervention was helpful, but controlled trials are needed to confirm these findings. ${ }^{11}$

Other studies have explored the integration of psychiatric care into post-conflict interventions. One report outlines the importance of integrating mental health into post-conflict rehabilitation, based on research done with Sierra Leonean and Liberian child soldiers. ${ }^{12}$ Similarly, Betancourt et al examined the cases of Russia and northern Uganda and found benefits to integrating psychiatric and psychosocial interventions.

Of the studies cited above about children in post-conflict settings, only one had any data about Liberia ${ }^{12}$, indicating how sparse the knowledge base is. To address this issue, we conducted a key informant needs assessment to characterize and quantify the mental health burden of the war on the children of Liberia. We asked key informants to tell us about the most emotionally disturbing events or experiences to have affected young Liberians in the past twenty years and how best to help specific vulnerable groups. A primary analysis of the quantitative data found that key informants' perceptions of the mental health needs of young people in Liberia are consistent with research on the harmful effects of war, violence and displacement on child and adolescent populations. Youth were perceived to be experiencing significant adverse emotional, behavioral and functional outcomes related to exposure to war and its aftermath. Appropriate treatment settings to target these issues were identified. (Borba C, Vesga Lopez O, Harris B, Gray D, Carney J, Dominguez S, et al. A Key Informant Survey on the Mental Health Needs of Children and Adolescents in PostConflict Liberia. Under review).
In the current study, we examine the findings of the qualitative questions that were part of the same survey. This data is based on open-ended questions regarding the types of interventions that would be most beneficial for different groups of vulnerable youth.

\section{Method}

\section{Study design and sample}

This research is a cross-sectional mixed methods survey conducted between March 30, 2009 and April 30, 2009 in Liberia with 171 local key Liberian informants. Researchers produced and analyzed the survey in their capacity as consultants to the Liberia Ministry of Health and Social Welfare. The Liberian Ministry of Health and Social Welfare of Liberia conducted the survey through funding from the McCall McBain Foundation. Quantitative portions of the survey were analyzed separately. ${ }^{13}$

There were 171 key informants who completed the survey. They were selected by the Ministry of Health and Social Welfare with input from local non-governmental organizations (NGOs). The participants were professionals or community health extenders - almost all Liberian (95\%) - in the health, education, religious, NGO, government and judicial sectors. Key informants were used because of the ethical issues associated with interviewing children and the possibility of identifying needs that could not be met due to limits of available care.

\section{Data Collection Procedures}

To standardize the survey collection, researchers (sociologists from a Liberian school of social work and a psychiatrist, psychologist and social worker from the Massachusetts General Hospital (MGH/Harvard Liberia Working Group)) trained Liberian interviewers to conduct the survey. The 21 data collectors were selected among students from the Mother Patern College of Health Sciences, School of Social Work in Liberia. The mean age of the interviewers was $40 \pm 12$ years with a range between 24 to 75 years. Five ( \%) reported having had another degree, such as an RN or BA. Training was conducted between February and March of 2009 which included classroom teaching followed by field observation. Training included instruction on survey administration and need for confidentiality and privacy. All key informant interviews were conducted in English, with interviewers asking questions verbally and writing the responses given by key informants. All surveys were reviewed for completeness and missing information. Researchers worked closely with interviewers to provide feedback and answer questions.

\section{Study Measures}

The survey instrument included questions on participants' socio-demographic characteristics such as age, gender, professional degrees, and nationality. The survey included qualitative questions on participants' ideas about what were the most disturbing events experienced by youth during the past 20 years. Participants were asked to answer separately for children ages 5-12 and adolescents and young adults ages 13-22. They were also asked about what would be the most effective interventions for helping different vulnerable groups of young people, including orphans, homeless children, former combatants and children of former combatants, 
mentally ill, persons with epilepsy, criminals, and violent youth. These questions were open-ended. For each case, participants were asked to name three traumatic events and three interventions.

\section{Ethics}

Ethical approval was obtained from the review board of the Liberian Ministry of Health and Social Welfare. Written informed consent was obtained from all participants. Every effort was made to ensure protection and confidentiality of the participants. Although participants' personal information was recorded for follow-up, any identifying information that linked the questionnaire with the response was removed prior to analysis. Participants did not receive any compensation for participating in the study. They were informed that participation and lack thereof would not affect them directly, and that they could withdraw consent or stop participating at anytime. Participants were explicitly given the right to refuse participation.

\section{Data Analysis}

This qualitative content analysis was performed by four coders who worked separately and sequentially. First, manifest content was grouped thematically. Thematic groupings were then labeled, and these group labels were used to generate broad themes. These broad, overarching themes were divided into sub-themes. Responses were tallied to determine the number that represented each theme and subtheme. Informants were separated by age and gender and results were analyzed separately for each category and collectively.

\section{Results}

Socio-demographic characteristics of the key informants are shown in Table I. Of the sample population of respondents $(\mathrm{N}=171)$, nearly two-thirds $(64.3 \%)$ were male. The average age was 43.8 years, \pm 9.5 years. The mean time a respondent reported living in Liberia was 37.3 years, \pm 14.9 years. The majority of respondents (64.9\%) lived in Montserrado County. The next most populous county was Bong, where $5.8 \%$ of the sample resided. The overwhelming majority of key informants were Liberian (94.7\%), with the remaining respondents reporting their nationality as other (1.8\%), American or British (1.2\% each), or Spanish or South African Dutch (0.6\% each). A large percentage of the respondents were in the health field sector (62.6\%). Only 1.8\% of the sample did not have a degree.

We found that while different interventions were preferred for different groups of young people, some interventions were mentioned for all groups and by all groups of key informants. These included counseling, education, and skills training. Also frequently chosen were housing, community reintegration, recreation, and medical care. The results for each group of young Liberians and each group of key informants are represented in Tables 1-IX. In general, key informants reported similar concerns regardless of their ages or genders. Notable exceptions to this were in interventions for youth who joined fighting forces. Men over 50 were the only ones to recommend apology and reparations. Similarly, in recommendations for criminals and violent youth, a number of men mentioned prison, whereas the women did not.

\begin{tabular}{|c|c|c|}
\hline \multicolumn{3}{|c|}{ Table I: Socio-Demographics of 171 Liberian Key Informants ${ }^{1}$} \\
\hline Characteristic & $N(\%$ of pop.) & Mean (SD) \\
\hline Age & & $43.8(9.5)$ \\
\hline Years in Liberia & & $37.3(14.9)$ \\
\hline $\begin{array}{l}\text { Sex } \\
\text { Male } \\
\text { Female }\end{array}$ & $\begin{array}{l}110(64) \\
61(36)\end{array}$ & \\
\hline $\begin{array}{l}\text { County } \\
\text { Montserrado } \\
\text { Bong } \\
\text { Grand Bassa } \\
\text { Lofa } \\
\text { Maryland } \\
\text { River Gee } \\
\text { Bomi } \\
\text { Grand Gedeh } \\
\text { Gbarpolu } \\
\text { Grand Cape Mount } \\
\text { Nimba } \\
\text { Sinoe } \\
\text { Grand Ku } \\
\text { Margibi } \\
\text { River Cress }\end{array}$ & $\begin{array}{l}111(64.9) \\
10(5.8) \\
8(4.7) \\
6(3.5) \\
6(3.5) \\
4(2.3) \\
4(2.3) \\
4(2.3) \\
3(1.8) \\
3(1.8) \\
3(1.8) \\
3(1.8) \\
3(1.8) \\
2(1.2) \\
1(0.6)\end{array}$ & \\
\hline $\begin{array}{l}\text { Nationality } \\
\text { Liberian } \\
\text { American } \\
\text { British } \\
\text { Spanish } \\
\text { South African Dutch } \\
\text { Other }\end{array}$ & $\begin{array}{l}162(94.7) \\
2(1.2) \\
2(1.2) \\
1(0.6) \\
1(0.6) \\
3(1.8)\end{array}$ & \\
\hline $\begin{array}{l}\text { Sectora } \\
\text { Health field worker }^{\text {}} \\
\text { Government employee } \\
\text { NGO/CSOc } \\
\text { Practicing cliniciand } \\
\text { Educator } \\
\text { Traditional Healer }\end{array}$ & $\begin{array}{l}107(62.6) \\
62(36.3) \\
66(38.6) \\
65(38.0) \\
37(21.6) \\
4(2.3)\end{array}$ & \\
\hline $\begin{array}{l}\text { Degree } \\
\text { Medical Doctor (MD) } \\
\text { Registered Nurse (RN) } \\
\text { Social Worker (BSW, AASW, MSW) } \\
\text { Physicians Assistant } \\
\text { Bachelors Degree } \\
\text { Masters Degree } \\
\text { Associates Degree } \\
\text { Certificate } \\
\text { High School Diploma } \\
\text { Certified Midwife } \\
\text { PhD } \\
\text { JD/LLN } \\
\text { University Student } \\
\text { Counselor } \\
\text { Other/Not Specified } \\
\text { None }\end{array}$ & $\begin{array}{l}15(8.8) \\
14(8.2) \\
20(11.7) \\
9(5.3) \\
41(24.0) \\
17(9.9) \\
6(3.5) \\
12(7.0) \\
2(1.2) \\
1(0.6) \\
1(0.6) \\
4(2.3) \\
2(1.2) \\
3(1.8) \\
21(12.3) \\
3(1.8)\end{array}$ & \\
\hline \multicolumn{3}{|c|}{$\begin{array}{l}\text { Notes: Due to rounding, percents may not equal } 100 \text {. } \\
\text { aPercent > } 100 \text { due to respondents working in multiple sectors } \\
\text { bIncludes all administrators and NGO workers who specified their } \\
\text { involvement with health related activities. It does not include all social } \\
\text { workers. } \\
\text { वncludes all UN and WHO employees } \\
\text { aRepresents those whose key role included clinical practice. It does not } \\
\text { represent all informants with a clinical degree. }\end{array}$} \\
\hline
\end{tabular}




\begin{tabular}{|c|c|c|c|c|c|c|}
\hline $\begin{array}{l}\text { Orphans } \\
\text { THEMES }\end{array}$ & $\begin{array}{l}F<40 \\
\text { SUB-THEMES }\end{array}$ & $\begin{array}{l}\text { F 40-49 } \\
\text { SUB-THEMES }\end{array}$ & $\begin{array}{l}\text { F50+ } \\
\text { SUB-THEMES }\end{array}$ & $\begin{array}{l}M<40 \\
\text { SUB-THEMES }\end{array}$ & $\begin{array}{l}\text { M 40-49 } \\
\text { SUB-THEMES }\end{array}$ & $\begin{array}{l}\text { M 50+ } \\
\text { SUB-THEMES }\end{array}$ \\
\hline Basic needs & $\begin{array}{l}\text { Housing } \\
\text { Food } \\
\text { Clothing }\end{array}$ & Housing & Housing & Housing & $\begin{array}{l}\text { Housing } \\
\text { Food } \\
\text { Clothing }\end{array}$ & $\begin{array}{l}\text { Housing } \\
\text { Food } \\
\text { Clothing }\end{array}$ \\
\hline Community & $\begin{array}{l}\text { Reunite families } \\
\text { Foster care } \\
\text { Orphanage }\end{array}$ & $\begin{array}{l}\text { Reunite families } \\
\text { Foster care } \\
\text { Orphanage }\end{array}$ & $\begin{array}{l}\text { Foster care } \\
\text { Orphanage } \\
\text { Loving care }\end{array}$ & $\begin{array}{l}\text { Foster care } \\
\text { Orphanage }\end{array}$ & $\begin{array}{l}\text { Foster care } \\
\text { Orphanage }\end{array}$ & $\begin{array}{l}\text { Reunite families } \\
\text { Orphanage }\end{array}$ \\
\hline Education & Academic & Academic & $\begin{array}{l}\text { Academic } \\
\text { Vocational }\end{array}$ & $\begin{array}{l}\text { Academic } \\
\text { Vocational } \\
\text { Life skills }\end{array}$ & $\begin{array}{l}\text { Academic } \\
\text { Vocational }\end{array}$ & $\begin{array}{l}\text { Academic } \\
\text { Life skills }\end{array}$ \\
\hline Health & Medical & Counseling & & Medical & $\begin{array}{l}\text { Medical } \\
\text { Counseling }\end{array}$ & Medical \\
\hline
\end{tabular}

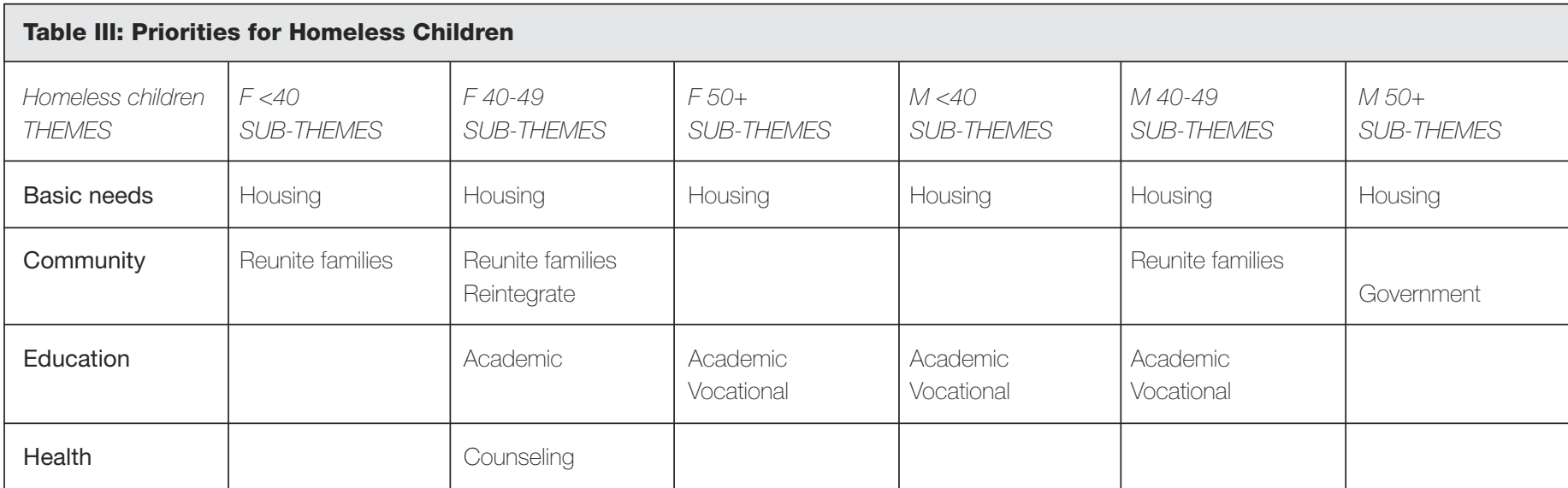

\section{Table IV: Priorities for Males who were Involved with Fighting Forces}

\begin{tabular}{|c|c|c|c|c|c|c|}
\hline $\begin{array}{l}\text { Male ex-combatants } \\
\text { THEMES }\end{array}$ & $\begin{array}{l}F<40 \\
\text { SUB-THEMES }\end{array}$ & $\begin{array}{l}\text { F } 40-49 \\
\text { SUB-THEMES }\end{array}$ & $\begin{array}{l}\text { F50+ } \\
\text { SUB-THEMES }\end{array}$ & $\begin{array}{l}M<40 \\
\text { SUB-THEMES }\end{array}$ & $\begin{array}{l}\text { M 40-49 } \\
\text { SUB-THEMES }\end{array}$ & $\begin{array}{l}\text { M 50+ } \\
\text { SUB-THEMES }\end{array}$ \\
\hline Community & & & & Reintegration & & \\
\hline Education & $\begin{array}{l}\text { Academic } \\
\text { Vocational } \\
\text { Job }\end{array}$ & $\begin{array}{l}\text { Academic } \\
\text { Vocational } \\
\text { Job }\end{array}$ & $\begin{array}{l}\text { Vocational } \\
\text { Job }\end{array}$ & $\begin{array}{l}\text { Academic } \\
\text { Vocational } \\
\text { Life skills } \\
\text { Jobs }\end{array}$ & $\begin{array}{l}\text { Academic } \\
\text { Vocational } \\
\text { Life skills } \\
\text { Jobs }\end{array}$ & $\begin{array}{l}\text { Academic } \\
\text { Vocational } \\
\text { Life skills } \\
\text { Jobs }\end{array}$ \\
\hline Health & Counseling & Counseling & Counseling & Counseling & $\begin{array}{l}\text { Counseling } \\
\text { Counseling }\end{array}$ & Counseling \\
\hline
\end{tabular}

\section{Table V: Priorities for Females who were Involved with Fighting Forces}

\begin{tabular}{|l|l|l|l|l|l|l|}
\hline $\begin{array}{l}\text { Female ex-combatants } \\
\text { THEMES }\end{array}$ & $\begin{array}{l}\text { F<40 } \\
\text { SUB-THEMES }\end{array}$ & $\begin{array}{l}\text { F 40-49 } \\
\text { SUB-THEMES }\end{array}$ & $\begin{array}{l}\text { F50+ } \\
\text { SUB-THEMES }\end{array}$ & $\begin{array}{l}\text { M<40 } \\
\text { SUB-THEMES }\end{array}$ & $\begin{array}{l}\text { M 40-49 } \\
\text { SUB-THEMES }\end{array}$ & $\begin{array}{l}\text { M 50+ } \\
\text { SUB-THEMES }\end{array}$ \\
\hline Community & $\begin{array}{l}\text { Academic } \\
\text { Civic } \\
\text { Vocational } \\
\text { Life skills }\end{array}$ & $\begin{array}{l}\text { Academic } \\
\text { Vocational } \\
\text { Life skills }\end{array}$ & $\begin{array}{l}\text { Vocatoinal } \\
\text { Life skills } \\
\text { Jobs }\end{array}$ & $\begin{array}{l}\text { Academic } \\
\text { Reproductive } \\
\text { Vocational } \\
\text { Life skills } \\
\text { Jobs }\end{array}$ & $\begin{array}{l}\text { Academic } \\
\text { Reproductive } \\
\text { Vocational } \\
\text { Life skills } \\
\text { Jobs }\end{array}$ & $\begin{array}{l}\text { Academic } \\
\text { Vocational } \\
\text { Life skills }\end{array}$ \\
\hline Health & Counseling & Counseling & Counseling & Counseling & Counseling & Counseling \\
\hline
\end{tabular}




\begin{tabular}{|l|l|l|l|l|l|l|}
\hline \multicolumn{2}{|l}{ Table V: Priorities for Children of Ex-combatants } \\
\hline $\begin{array}{l}\text { Children of ex-combatants } \\
\text { THEMES }\end{array}$ & $\begin{array}{l}\text { F }<40 \\
\text { SUB-THEMES }\end{array}$ & $\begin{array}{l}\text { F 40-49 } \\
\text { SUB-THEMES }\end{array}$ & $\begin{array}{l}\text { F50+ } \\
\text { SUB-THEMES }\end{array}$ & $\begin{array}{l}\text { M<40 } \\
\text { SUB-THEMES }\end{array}$ & $\begin{array}{l}\text { M 40-49 } \\
\text { SUB-THEMES }\end{array}$ & $\begin{array}{l}\text { M 50+ } \\
\text { SUB-THEMES }\end{array}$ \\
\hline Community & $\begin{array}{l}\text { Reunite families } \\
\text { Reintegrate }\end{array}$ & Caregiver support & Reintegrate & Reintegrate & Caregiver support & Reintegrate \\
\hline Education & Academic & $\begin{array}{l}\text { Academic } \\
\text { Vocational }\end{array}$ & Academic & $\begin{array}{l}\text { Academic } \\
\text { Vocational } \\
\text { Jobs }\end{array}$ & $\begin{array}{l}\text { Academic } \\
\text { Vocational } \\
\text { Life skills } \\
\text { Jobs } \\
\text { Training for parents }\end{array}$ & $\begin{array}{l}\text { Academic } \\
\text { Vocational } \\
\text { Trabs }\end{array}$ \\
\hline Health & Counseling for parents \\
\hline
\end{tabular}

Table VII: Priorities for Severely Mentally III Children

\begin{tabular}{|l|l|l|l|l|l|l|}
$\begin{array}{l}\text { Severely mentally ill } \\
\text { THEMES }\end{array}$ & $\begin{array}{l}\text { F }<40 \\
\text { SUB-THEMES }\end{array}$ & $\begin{array}{l}\text { F 40-49 } \\
\text { SUB-THEMES }\end{array}$ & $\begin{array}{l}\text { F50+ } \\
\text { SUB-THEMES }\end{array}$ & $\begin{array}{l}\text { M }<40 \\
\text { SUB-THEMES }\end{array}$ & $\begin{array}{l}\text { M 40-49 } \\
\text { SUB-THEMES }\end{array}$ & $\begin{array}{l}\text { M 50+ } \\
\text { SUB-THEMES }\end{array}$ \\
\hline Health & $\begin{array}{l}\text { Institutionalize } \\
\text { Counseling } \\
\text { Treatment }\end{array}$ & $\begin{array}{l}\text { Clinics } \\
\text { Medication } \\
\text { Treaters }\end{array}$ & $\begin{array}{l}\text { Institutionalize } \\
\text { Counseling } \\
\text { Treatment }\end{array}$ & $\begin{array}{l}\text { Professionals } \\
\text { Clinic } \\
\text { Treatment }\end{array}$ & $\begin{array}{l}\text { Clinic Counseling } \\
\text { Treatment }\end{array}$ & $\begin{array}{l}\text { Clinic } \\
\text { Government } \\
\text { Professionals }\end{array}$ \\
\hline
\end{tabular}

Table VIII: Priorities for Children with Epilepsy

\begin{tabular}{|l|l|l|l|l|l|l|}
\hline $\begin{array}{l}\text { Children with epilepsy } \\
\text { THEMES }\end{array}$ & $\begin{array}{l}\text { F }<40 \\
\text { SUB-THEMES }\end{array}$ & $\begin{array}{l}\text { F 40-49 } \\
\text { SUB-THEMES }\end{array}$ & $\begin{array}{l}\text { F50+ } \\
\text { SUB-THEMES }\end{array}$ & $\begin{array}{l}\text { M }<40 \\
\text { SUB-THEMES }\end{array}$ & $\begin{array}{l}\text { M 40-49 } \\
\text { SUB-THEMES }\end{array}$ & $\begin{array}{l}\text { M 50+ } \\
\text { SUB-THEMES }\end{array}$ \\
\hline Community & Awareness & Awareness & $\begin{array}{l}\text { Awareness } \\
\text { Institutionalize }\end{array}$ & Awareness & Awareness & Awareness \\
\hline Education & Illness & Medical & $\begin{array}{l}\text { Medication } \\
\text { Counseling }\end{array}$ & Medication & $\begin{array}{l}\text { Medical } \\
\text { Medication }\end{array}$ & $\begin{array}{l}\text { Medical } \\
\text { Medication }\end{array}$ \\
\hline
\end{tabular}

\section{Table IX: Priorities for those Convicted of Crimes}

\begin{tabular}{|c|c|c|c|c|c|c|}
\hline $\begin{array}{l}\text { Criminals } \\
\text { THEMES }\end{array}$ & $\begin{array}{l}F<40 \\
\text { SUB-THEMES }\end{array}$ & $\begin{array}{l}\text { F } 40-49 \\
\text { SUB-THEMES }\end{array}$ & $\begin{array}{l}\text { F50+ } \\
\text { SUB-THEMES }\end{array}$ & $\begin{array}{l}M<40 \\
\text { SUB-THEMES }\end{array}$ & $\begin{array}{l}\text { M 40-49 } \\
\text { SUB-THEMES }\end{array}$ & $\begin{array}{l}\text { M 50+ } \\
\text { SUB-THEMES }\end{array}$ \\
\hline Basic needs & Food & & & & & \\
\hline Community & & Rehab & & Safety & Legal & Rehab \\
\hline Education & $\begin{array}{l}\text { Vocational } \\
\text { Civic }\end{array}$ & Vocational & $\begin{array}{l}\text { Vocational } \\
\text { Civic }\end{array}$ & $\begin{array}{l}\text { Vocational } \\
\text { Life skills }\end{array}$ & Legal & $\begin{array}{l}\text { Vocational } \\
\text { Academic } \\
\text { Civic }\end{array}$ \\
\hline Health & Counseling & Counseling & Counseling & Counseling & Counseling & Counseling \\
\hline
\end{tabular}

\section{Table X: Priorities for Violent Youth}

\begin{tabular}{|c|c|c|c|c|c|c|}
\hline $\begin{array}{l}\text { Violent youth } \\
\text { THEMES }\end{array}$ & $\begin{array}{l}F<40 \\
\text { SUB-THEMES }\end{array}$ & $\begin{array}{l}\text { F } 40-49 \\
\text { SUB-THEMES }\end{array}$ & $\begin{array}{l}\text { F50+ } \\
\text { SUB-THEMES }\end{array}$ & $\begin{array}{l}M<40 \\
\text { SUB-THEMES }\end{array}$ & $\begin{array}{l}\text { M 40-49 } \\
\text { SUB-THEMES }\end{array}$ & $\begin{array}{l}\text { M 50+ } \\
\text { SUB-THEMES }\end{array}$ \\
\hline Community & & & Rehabilitation & & & \\
\hline Education & & $\begin{array}{l}\text { Vocational } \\
\text { Life skills } \\
\text { Recreation }\end{array}$ & $\begin{array}{l}\text { Vocational } \\
\text { Recreation }\end{array}$ & $\begin{array}{l}\text { Vocational } \\
\text { Recreation }\end{array}$ & $\begin{array}{l}\text { Academic } \\
\text { Vocational } \\
\text { Civic }\end{array}$ & $\begin{array}{l}\text { Academic } \\
\text { Vocational } \\
\text { Recreation }\end{array}$ \\
\hline Health & Counseling & Counseling & Counseling & Counseling & Counseling & Counseling \\
\hline
\end{tabular}




\section{Orphans and homeless children}

The question of where to place these children and who is responsible for them was a consistent theme. Some emphasized placement in families, while others favored creating a home for them. One woman suggested, "Create a network of families [and] give them compensation." Another offered, "Support orphanages that meet international standards and monitor them." Education was consistently among the top priorities reported across age and gender. Housing was also mentioned consistently. While men talked explicitly about housing ("Get them off the street"), women tended to talk about housing, safety and care together, and suggested either foster care or orphanages ("Create places where they feel safe to interact with adults"). Men mentioned shelter as a separate need and then also talked about family reunification, foster care, and orphanages. All ages and genders mentioned both orphanages and foster care with similar frequency. Key informants from each age and gender group mentioned counseling or psychological support, but it did not rank among the top priorities. Men of all ages mentioned medical care, but only women under 40 did. The need for government support for the care of these children was consistently mentioned by several people in each group.

Housing was the top priority among all groups for these youngsters. Other basic needs were also mentioned. Education and skills training was also mentioned across the board.

Reuniting children with their families and reintegrating them into the community were both mentioned consistently. Reunification was mentioned by all groups; reintegration was mentioned more by those under 50. Counseling and psychological support were mentioned by all groups but more by men and women in their 40s. All mentioned the need for government support, but for men over 50, this was a top priority.

\section{Ex combatants and children of ex combatants}

For former child soldiers, the question was raised as to whether they owe some sort of reparations to the community. Vocational skills, education, and jobs, in that order, were consistently given high priority for these youngsters. Among women in their 40s and men of all ages, skills training was the most frequently cited need. Women under 40 and over 50 mentioned counseling more often than skills, but skills training was second. Resocialization and demobilization were mentioned by nine women and 42 men. Most common were comments about the importance of reducing stigma and facilitating acceptance back into society, like this one: "Rehabilitation through school program[s], community-based psychosocial activities." A few comments by men over 50 emphasized the importance of making some form of reparations and seeking forgiveness: "Counseling to help children to [apologize] (admit guilt) to communities they hurt."

Similarly, counseling and job skills were most frequently mentioned for female ex-combatants, as for males. Women in their 40s and men over 50 mentioned counseling most, while other groups mentioned skills more. Education was also mentioned frequently, including civic education about the law and legal rights, and sex education and access to contraception. There was frequent mention of the risk of sexual exploitation and the need to provide these girls with skills so they do not fall prey to this. This concern was mentioned by all groups but more frequently by men. Social reintegration and destigmatization was cited by all groups. Three women and four men mentioned demobilization and education in non-violence. Only one man over 50 mentioned the need for an apology.

Counseling and education were among the most frequently mentioned interventions by all groups for children of excombatants. Counseling was the most cited need by women under 40 and women of unknown age. Education was top for other groups. Support for caregivers was mentioned by women in their 40s and men of all ages. It is unclear if this referred to the ex-combatants themselves, who are the parents, or if it was assumed that the children were being cared for by others in the community. Community acceptance and reintegration were mentioned consistently, as were basic needs. Loans for education and entrepreneurship were mentioned by women over 50 and men of all ages.

\section{Mentally ill}

For mentally-ill children, the question emerged as to whether they would be best served by reintegration or institutionalization and whether traditional or Western approaches to treatment would be most effective. "Provide facilities for treatment" was a sentiment shared by all groups. This was the most often cited need among all groups, except men under 40, who gave most emphasis to the need for trained mental health professionals. Counseling and medication were mentioned with nearly equal frequency by all groups. Most needs revolved around medical care. Basic needs and education were mentioned only a few times by each group. Community awareness and stigma reduction received a few mentions, as did caregiver support. Men in each age group, but no women, mentioned the need for decentralization of care so that it is accessible in rural areas.

\section{Children with epilepsy}

For children with epilepsy, medical care and medication were most frequently mentioned by all groups. There was some mention of the need for trained professionals, but less than for the previous group. A few people in each group cited the importance of community awareness, stigma reduction, and caregiver support. Interestingly, men under 40 were most likely to mention caregiver support. Men over 50 made a few erroneous statements about epilepsy, for example that these children are "mentally retarded" or exhibit "psychotic behavior."

\section{Criminals}

For those in the criminal justice system, responses ranged from those who recommended locking them up to protecting them and guaranteeing their rights, to educating them and providing skills. Some placed the blame for the behavior on the kids while others felt that it was the responsibility of the family and community to help and support them. Civic education so they have a clear understanding of rules and consequences: "Training for young people in human rights and to respect the law in our community."

Counseling and skills training were consistently mentioned for this group. Men also mentioned legal themes. Responses ranged from the importance of protecting children's legal rights to the need for justice to be carried out fairly and swiftly, to the need to punish offenders and imprison them for the protection of society ("They always want to escape punishment"). 


\section{Violent youth}

Similar themes emerged when people were asked specifically about violent youth. Again, there was a tension between protecting them and protecting society from them. Again, though, the same needs were mentioned as for all other young people; basic needs, recreation, education, skills, and jobs. Counseling was consistently the most frequently cited need for this population. Recreation, skills, and education were also mentioned frequently, as well as "rehabilitation," undefined. Several men in each age group mentioned prison, but none of the women did.

\section{Discussion}

This study explored the perceived mental health needs of young people in Liberia by 171 local key informants in the health, education, NGO, religious and government sectors. The objective was to understand local stakeholders' perceived notions of the impact of violence on young people and the priority mental health needs of young Liberians. Among key informants, there was a consensus that all groups of vulnerable young people need access to medical care, mental health care and education.

This study was the first of its kind to focus on the needs in Liberia specifically. Whereas other studies assessed the efficacy of specific interventions, this study asked which types of interventions should be undertaken. Nevertheless, many of our findings are consistent with other research findings in analogous settings. A key informant study done in Rwanda identified perseverance, self esteem, family unity, good parenting, and community cohesion as protective factors for children who experienced trauma. ${ }^{4}$ Similarly, key informants in our study believed that family reunification and community reintegration were important interventions to support young people traumatized by war. Other studies have demonstrated the importance of caregivers and a sense of relative security and predictability to long term outcomes of children in postconflict settings. ${ }^{7-9}$ Some studies have also tested brief educational interventions with promising results. ${ }^{10,11}$ This study similarly found that people believed that these types of interventions were needed, as well as suggesting other types of interventions that have not been widely studied in postconflict youth.

While the majority of respondents were interested in helping these vulnerable youth populations, there were notable exceptions. Some respondents, generally older men, were more interested in punishing the youth for acts committed during the war and protecting their communities from perceived risk of future violence. It will be important to further characterize the views of this group in order to anticipate how interventions will be received by different segments of the population and where gaps in awareness and understanding of mental illness need to be addressed.

This study had a number of limitations. It used key informants selected by the government ministries and residing in Monrovia. Many of them work with children as professionals in the community, but some do not, and most have no experience in mental health. A different segment of the population might have a different set of priorities for child and adolescent mental health, and the children themselves would likely have yet another perspective. Interviewers were used rather than having key informants complete the surveys themselves. This was done in order to ensure that they questions were understood as they were written. However, subjects may have felt pressure to give socially acceptable answers and may have altered their responses accordingly. Even with this provision, some responses indicated that some subjects did not understand some questions.

\section{Conclusion}

Our findings suggest that the needs of post-conflict Liberian youth span a variety of domains, including physical, emotional, medical, psychological, and educational. Most of our respondents favor community-based interventions as well as assistance from trained professionals. It will therefore be important to develop and support programs based in schools and homes, as well as training teachers, physicians, and other mental health professionals committed to serving the local population. Designing interventions that the Liberian people believe will be effective is critical, as well as remaining open to other needs and approaches that may not have been captured by this initial key informant survey.

\section{References}

1. Liberia Institute of Statistics and Geo-Information Services (LISGIS). 2008 Population and Housing Census. 2009.

2. UNDP. National Human Development Report of Liberia2006.

3. Attanayake V, McKay R, Joffres M, Singh S, Burkle F, Jr., Mills E. Prevalence of mental disorders among children exposed to war: a systematic review of 7,920 children. Med Confl Surviv 2009 ;25(1):4-19.

4. Betancourt TS, Meyers-Ohki S, Stulac SN, Elizabeth Barrera A, Mushashi C, Beardslee WR. Nothing can defeat combined hands (Abashize hamwe ntakibananira): Protective processes and resilience in Rwandan children and families affected by HIVIAIDS. Soc Sci Med 201 1; 73(5):693-701.

5. Kruk ME, Rockers PC, Williams EH, Varpilah ST, Macauley R, Saydee G, et al. Availability of essential health services in postconflict Liberia. Bull World Health Organ 2010 ;88(7):527-34.

6. Miller KE, Rasmussen A. War exposure, daily stressors, and mental health in conflict and post-conflict settings: bridging the divide between trauma-focused and psychosocial frameworks. Soc Sci Med $2010 ; 70(1): 7-16$.

7. Betancourt TS, Agnew-Blais J, Gilman SE, Williams DR, Ellis BH. Past horrors, present struggles: the role of stigma in the association between war experiences and psychosocial adjustment among former child soldiers in Sierra Leone. Soc Sci Med 2010;70(1):17-26.

8. Antonovsky H, Sagy S. The development of a sense of coherence and its impact on responses to stress situations. The Journal of Social Psychology 1986 ;126(2):213-25.

9. Ebina R, Yamazaki Y. Sense of coherence and coping in adolescents directly affected by the 1991--5 war in Croatia. Promot Educ 2008 ;15(4):5-10.

10. Ager A, Akesson B, Stark L, Flouri E, Okot B, McCollister F, et al. The impact of the school-based Psychosocial Structured Activities (PSSA) program on conflict-affected children in northern Uganda.J Child Psychol Psychiatry 2011; 52(11):1124-1133.

11. Gupta L, Zimmer C. Psychosocial intervention for war-affected children in Sierra Leone. Br J Psychiatry 2008 ; 192(3):212-6.

12. Medeiros E. Integrating mental health into post-conflict rehabilitation: the case of Sierra Leonean and Liberian 'child soldiers'. J Health Psychol 2007 ;12(3):498-504. 